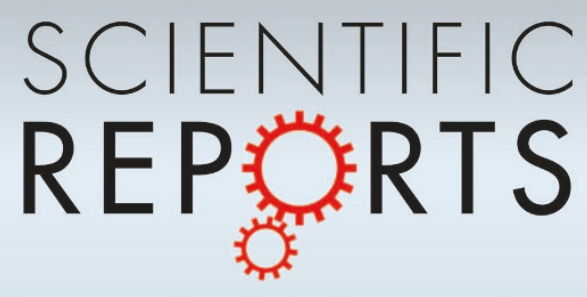

OPEN

SUBJECT AREAS:

PHASE TRANSITIONS

AND CRITICAL

PHENOMENA

ELECTRONIC PROPERTIES AND

MATERIALS

RAMAN SPECTROSCOPY

FERROELECTRICS AND MULTIFERROICS

Received

7 August 2014

Accepted

2 September 2014

Published

19 September 2014

Correspondence and requests for materials should be addressed to R.H. (rui.he@uni.edu) orJ.Z. (jmzhao@iphy. ac.cn)

\section{Double Charge Ordering States and Spin Ordering State Observed in a $\mathrm{RFe}_{2} \mathrm{O}_{4}$ System}

\author{
Fei Sun', Rui Wang' ', C. Aku-Leh³ , H. X. Yang' ${ }^{1}$ Rui He² \& Jimin Zhao' \\ 'Beijing National Laboratory for Condensed Matter Physics and Institute of Physics, Chinese Academy of Sciences, Beijing 100190 \\ China, ${ }^{2}$ Department of Physics, University of Northern lowa, Cedar Falls, lowa 50614, USA, ${ }^{3}$ ISciences, Ann Arbor, Michigan, \\ 48103, USA.
}

Charge, spin, and lattice degrees of orderings are of great interest in the layered quantum material $\mathrm{RFe}_{2} \mathrm{O}_{4}$ $(\mathrm{R}=\mathrm{Y}, \mathrm{Er}, \mathrm{Yb}, \mathrm{Tm}$, and $\mathrm{Lu})$ system. Recently many unique properties have been found using various experimental methods. However so far the nature of the two-dimensional (2D) charge ordering (CO) state is not clear and no observation of its fine structure in energy has been reported. Here we report unambiguous observation of double $2 \mathrm{D} \mathrm{CO}$ states at relatively high temperature in a polycrystalline $\mathrm{Er}_{0.1} \mathrm{Yb}_{0.9} \mathrm{Fe}_{2} \mathrm{O}_{4}$ using Raman scattering. The energy gaps between the $3 \mathrm{D}$ and the double $2 \mathrm{D}$ states are $170 \mathrm{meV}(41.2 \mathrm{THz})$ and $193 \mathrm{meV}(46.6 \mathrm{THz})$, respectively. We also observed a spin ordering (SO) state at below $210 \mathrm{~K}$ with characteristic energy of $45 \mathrm{meV}(10.7 \mathrm{THz})$. Our investigation experimentally identified new fine structures of quantum orders in the system, which also extends the capability of optical methods in investigating other layered quantum materials.

U nique properties based on various orderings in complex materials, especially the $\mathrm{RFe}_{2} \mathrm{O}_{4}$ system, have drawn great interest recently ${ }^{1-21}$. Experiments such as $\mathrm{x}$-ray diffraction ${ }^{1-2}$, neutron scattering ${ }^{4,13}$, electronic transport measurement ${ }^{8}$, in situ cooling transmission electron microscopy ${ }^{23}$, optical spectroscopy ${ }^{6,17}$, etc. have been carried out and illustrated various types of orderings and properties such as charge ordering ${ }^{5,9}$, spin ordering $^{1,5}$, giant magnetocapacitance ${ }^{9}$, giant room-temperature magnetodielectric response ${ }^{10}$, giant magnetic anisotropy ${ }^{20}$, etc in the system. However, there are very few reports on the detailed energy structure of the 2D CO states and optical methods have been less reported in identification of the spin ordering state. In this paper we show clear evidence of observing non-degenerate double $2 \mathrm{D}$ CO states and a ferrimagnetic SO state in an electronic ferroelectric material $\mathrm{Er}_{0.1} \mathrm{Yb}_{0.9} \mathrm{Fe}_{2} \mathrm{O}_{4}$ by using temperature-dependent Raman scattering. Furthermore, we have also observed two infrared-active phonon modes besides the full spectrum of Ramanactive phonon modes in it. This is a material with which we had for the first time observed the Stark effect in a solid, which confirmed the existence of strong local field and provided a spectroscopic evidence of the ferroelectric nature of the material ${ }^{7}$.

The $\mathrm{Er}_{0.1} \mathrm{Yb}_{0.9} \mathrm{Fe}_{2} \mathrm{O}_{4}$ crystal belongs to the $\mathrm{RFe}_{2} \mathrm{O}_{4}$ type mixed-valence materials, which has been known exhibiting $\mathrm{CO}$ composing $\mathrm{Fe}^{2+}$ and $\mathrm{Fe}^{3+}$ ions on a geometrically frustrated triangular lattice. The bulk ferroelectric polarization thus formed (i.e. electronic ferroelectricity) arises from the $3 \mathrm{D}$ alternating arrangement of valence-charges, instead of the spatial displacement of cations as in the conventional ferroelectric materials ${ }^{1,23}$. Among the many investigation methods, Raman and infrared (IR) spectroscopy investigations have been carried out on $\mathrm{LuFe}_{2} \mathrm{O}_{4}{ }^{6,16-18}$, illustrating its structural, magnetic and charge ordering properties. In the IR investigation a transition between the $2 \mathrm{D}$ and the $3 \mathrm{D} \mathrm{CO}$ was observed ${ }^{17}$. However, so far Raman and IR results have not provided any information on spin ordering in the $\mathrm{RFe}_{2} \mathrm{O}_{4}$ system, although neutron diffraction have been used to find the ferrimagnetic order in $\mathrm{LuFe}_{2} \mathrm{O}_{4}{ }^{22}$.

In this Article, a temperature-dependent Raman scattering study on the phase transition in polycrystalline $\mathrm{Er}_{0.1} \mathrm{Yb}_{0.9} \mathrm{Fe}_{2} \mathrm{O}_{4}$ has been carried out. Unlike available reports on Raman scattering and other optical spectroscopy of the $\mathrm{RFe}_{2} \mathrm{O}_{4}$ system, our experiment presents a full spectrum of the excitations and show five distinct new modes. Significantly we observed two non-degenerate $2 \mathrm{D}$ CO modes at the temperature range of $300 \sim 400 \mathrm{~K}$. We directly obtained the energy gaps between these $2 \mathrm{D}$ double CO states and the 3D CO state. Also we have observed one mode attributed to the ferrimagnetic spin ordering at below $210 \mathrm{~K}$. Furthermore two IR-active modes have also been identified due to the breakdown of inversion symmetry at the crystalline domain 


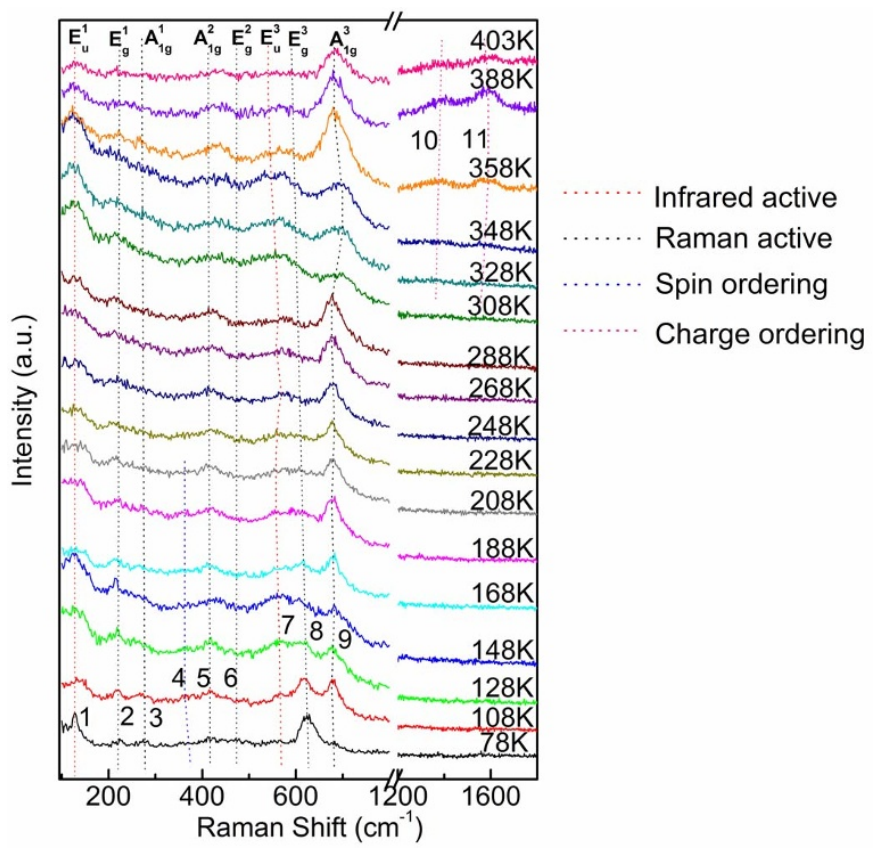

Figure 1 Raman spectra of $\mathrm{Er}_{0.1} \mathrm{Yb}_{0.9} \mathrm{Fe}_{2} \mathrm{O}_{4}$ at various temperatures. Raman peaks are numbered and highlighted by dashed lines.

boundaries. Our investigation extends or demonstrates the Raman (and optical) investigation capability in identifying the phase transitions and various orderings in such complex quantum materials.

\section{Results}

Variable-temperature Raman spectra of our polycrystalline $\mathrm{Er}_{0.1} \mathrm{Yb}_{0.9} \mathrm{Fe}_{2} \mathrm{O}_{4}$ are shown in Fig. 1. The temperature dependences of the frequencies and intensities of all the observed modes are shown in Figs. 2 and 3, respectively. We observed 11 modes altogether, of which the information is summarized in Table 1.

Significantly, two high frequency modes (numbered 10 and 11 in Figs. 1-3) begin to emerge when the temperature $T$ is raised to higher than $300 \mathrm{~K}$. These two Raman peaks cannot be assigned to higherorder Raman or IR modes since they appear only when $T$ is higher than $300 \mathrm{~K}$. Various diffraction techniques and theoretical analysis have been applied to the $\mathrm{LuFe}_{2} \mathrm{O}_{4}$ systems, which suggested a 3D CO to $2 \mathrm{D} \mathrm{CO}$ transition that occurs at a similar temperature when temperature is raised ${ }^{5,24}$. Here we assign these two high frequency modes to the $2 \mathrm{D}$ CO states in the $\mathrm{Er}_{0.1} \mathrm{Yb}_{0.9} \mathrm{Fe}_{2} \mathrm{O}_{4}$ crystal. The energy scale of these two modes is at the same order as those observed in other $\mathrm{RFe}_{2} \mathrm{O}_{4}$ systems using other methods ${ }^{3,25}$ (see below discussion). In light of the relatively high frequencies of these two modes we speculate that these two modes are due to in-plane interactions, which is relatively stronger due to closer distance between atoms and consistent with the 2D nature of the CO state.

As expected (see Discussions) all six Raman-active modes (with subscripts $g$ ) are observed (highlighted by black dashed lines in Fig. 1 and gray points in Fig. 2). Among them, the three Raman modes of $A_{1 g}$ symmetry are due to the out-of-plane vibrations (where atoms move perpendicularly to the $\mathrm{Yb}-\mathrm{O}, \mathrm{Er}-\mathrm{O}$, and $\mathrm{Fe}-\mathrm{O}$ planes, respectively), and the three $E_{\mathrm{g}}$ symmetry Raman modes corresponds to the in-plane vibrations ${ }^{6}$. A pair of modes (modes 1 and 7) exist in the entire range of temperature (highlighted by red dashed lines in Fig. 1 and red points in Fig. 2), whose frequencies are close to those of the $\mathrm{E}_{\mathrm{u}}{ }^{1}$ and $\mathrm{E}_{\mathrm{u}}{ }^{3}$ vibrations in the $\mathrm{RFe}_{2} \mathrm{O}_{4}$ type crystals ${ }^{6}$. We assign these two modes to the IR-active modes with $\mathrm{E}_{\mathrm{u}}{ }^{1}$ and $\mathrm{E}_{\mathrm{u}}{ }^{3}$ symmetries, respectively. Usually the IR active modes are not expected in Raman measurements for this material. The observation of the $\mathrm{E}_{\mathrm{u}}{ }^{1}$ and $\mathrm{E}_{\mathrm{u}}{ }^{3}$ symmetry modes can be well understood based on the poly-

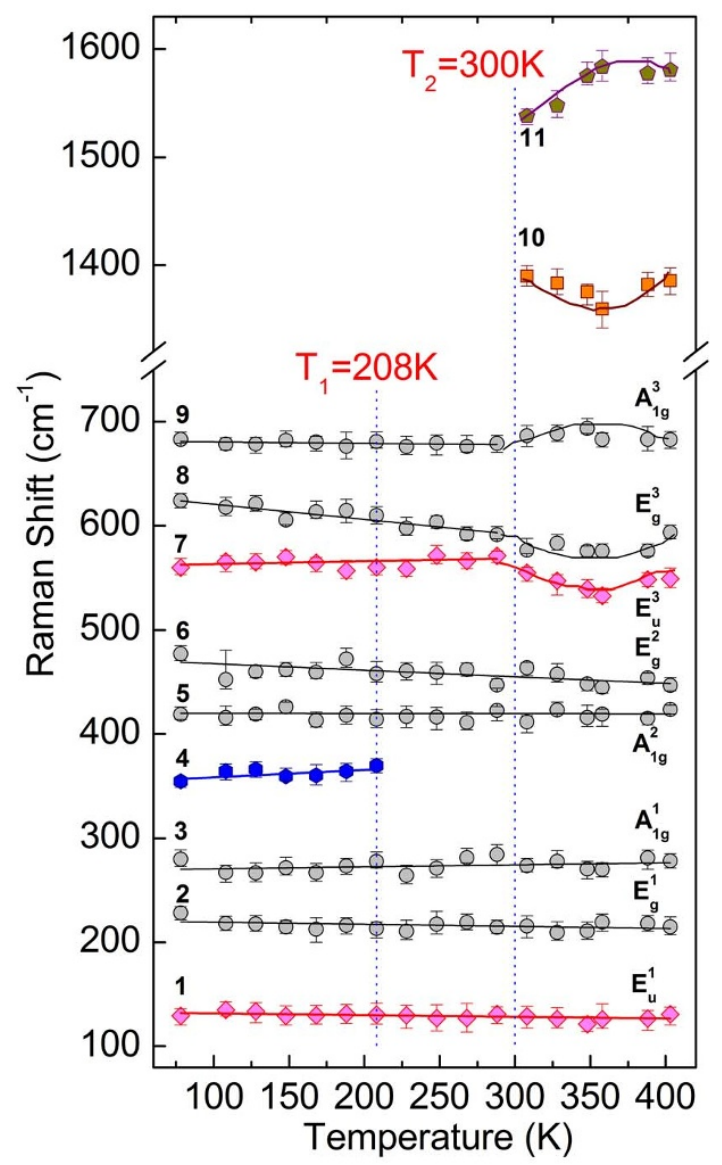

Figure $2 \mid$ Frequency of the 11 Raman modes highlighted in Fig. 1 as a function of temperature. The vertical dashed lines highlight the temperatures at which phase transitions occur. The solid lines are guides to the eye.

crystalline nature of the sample. The rich boundaries and vacancies in the sample break down the inversion symmetry of the crystal at the surfaces and the phonon parity selection rule is largely relaxed in our polycrystalline sample.

In Fig. 2 we can see that the frequencies of three Raman-active modes $\mathrm{E}_{\mathrm{g}}{ }^{1}, \mathrm{~A}_{1 \mathrm{~g}}{ }^{1}, \mathrm{~A}_{1 \mathrm{~g}}{ }^{2}$ and the lowest energy IR-active mode $\mathrm{E}_{\mathrm{u}}{ }^{1}$ do not show much variation in the entire temperature range. The $\mathrm{E}_{\mathrm{g}}{ }^{2}$ mode softens from $477 \mathrm{~cm}^{-1}$ to $447 \mathrm{~cm}^{-1}$ when $T$ changes from $78 \mathrm{~K}$ to $403 \mathrm{~K}$. The frequencies of the three higher energy vibrational modes $\mathrm{E}_{\mathrm{u}}{ }^{3}, \mathrm{E}_{\mathrm{g}}{ }^{3}$, and $\mathrm{A}_{1 \mathrm{~g}}{ }^{3}$ show non-monotonic dependence on $T$. When $T$ varies from $78 \mathrm{~K}$ to $300 \mathrm{~K}$, the $\mathrm{E}_{\mathrm{u}}{ }^{3}$ vibration hardens slightly, whereas the $\mathrm{E}_{\mathrm{g}}{ }^{3}$ vibration redshifts from $626 \mathrm{~cm}^{-1}$ to $593 \mathrm{~cm}^{-1}$. The $\mathrm{A}_{1 \mathrm{~g}}{ }^{3}$ vibrational frequency remains unchanged in this temperature range. For $T>300 \mathrm{~K}$, the $\mathrm{E}_{\mathrm{u}}{ }^{3}$ and $\mathrm{E}_{\mathrm{g}}{ }^{3}$ vibrational frequencies reach a minimum at about $350 \mathrm{~K}$, and the $\mathrm{A}_{1 \mathrm{~g}}{ }^{3}$ frequency reaches a maximum at $T \sim 350 \mathrm{~K}$. The spin-ordered mode 4 blueshifts slightly when $T$ increases from $78 \mathrm{~K}$ to $\sim 210 \mathrm{~K}$, and it disappears for $T$ higher than $210 \mathrm{~K}$. The two highest frequency modes (numbered 10 and 11) only appear when $T$ is higher than $300 \mathrm{~K}$. The frequency of mode 10 has a minimum value at $T \sim 350 \mathrm{~K}$, whereas the frequency of mode 11 has a maximum value at $T \sim 350 \mathrm{~K}$.

In Fig. 3 we show that the intensities of modes 1-3 and 5-7 do not display significant changes for $T<300 \mathrm{~K}$. The intensity of mode 8 decreases when $T$ changes from $78 \mathrm{~K}$ to $210 \mathrm{~K}$. Between $210 \mathrm{~K}$ and $300 \mathrm{~K}$, the intensity of mode 8 does not change. Specifically, at around $150 \mathrm{~K}$, the mode intensity decreases and a transfer of oscillator strength occurs the surrounding background gains weight. For mode number 9 , its intensity changes when $T$ changes from $78 \mathrm{~K}$ to 


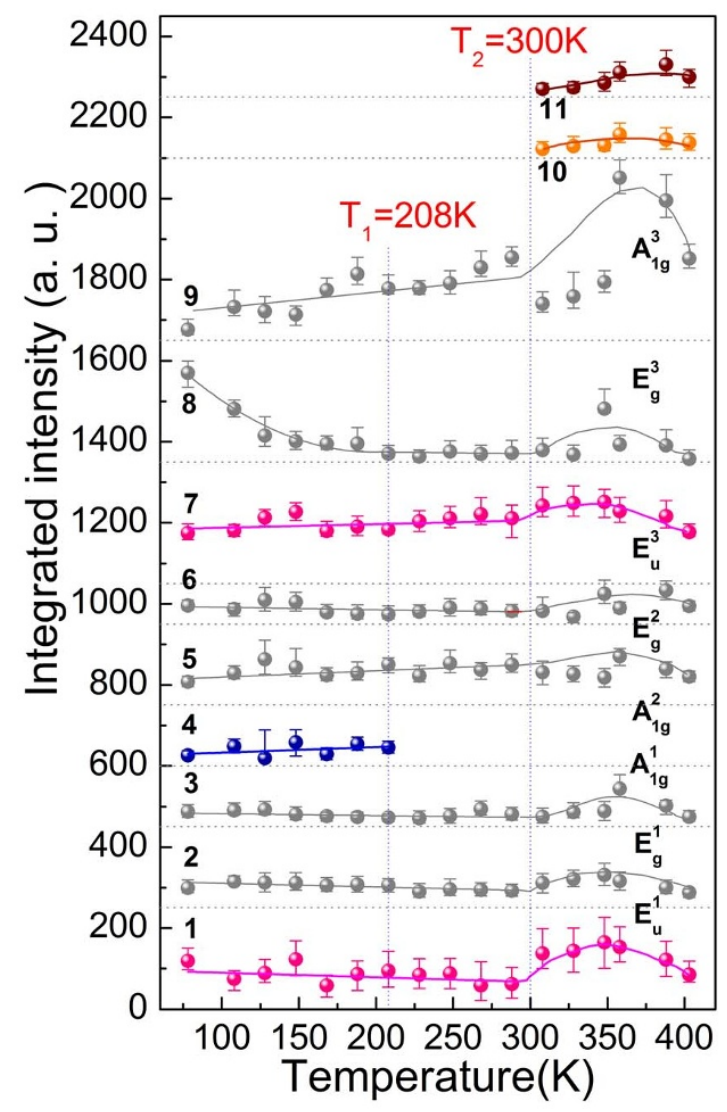

Figure $3 \mid$ Integrated intensities of the 11 Raman modes (see Fig. 1) as a function of temperature. The vertical dashed lines highlight the temperatures at which phase transitions occur. The intensity for each mode is shifted vertically for clarity. The solid curves are guides to the eye. The horizontal dotted lines below each solid curve represent the base line of intensity without offsets.

$300 \mathrm{~K}$. All modes (modes 1 to 11 except mode 4) show their strongest intensities at $T \sim 350 \mathrm{~K}$. The intensity of mode 4 which we attribute to spin ordering does not show noticeable changes for the temperature range of $78 \mathrm{~K} \sim 210 \mathrm{~K}$.

\section{Discussion}

Charge ordering phase transition. Since Fe ion has an average valence of $2.5+$, equal numbers of $\mathrm{Fe}^{2+}$ and $\mathrm{Fe}^{3+}$ ions co-exist in a lattice unit cell. Compared to the average valence of $2.5+$ for the Fe ions, the $\mathrm{Fe}^{2+}$ and $\mathrm{Fe}^{3+}$ ions are practically equivalent to possessing a $0.5-$ and $0.5+$ valence, respectively. In the triangular lattice charge frustration between the 0.5 - and $0.5+$ ions is thus inevitable, which leads to various forms of charge orders. Most noticeably charge order phase transition has been confirmed by various scattering experiments, such as X-ray scattering, neutron scattering, electron diffraction, etc $c^{2-3,22,26-27}$. For example, $\mathrm{LuFe}_{2} \mathrm{O}_{4}$ exhibits a phase transition at $330 \mathrm{~K}$, where a $2 \mathrm{D} \mathrm{CO}$ emerges and replaces the low temperature 3D CO and persists up to $500 \mathrm{~K}^{2,28}$. It is speculated that the $\mathrm{Fe}^{2+}$ and $\mathrm{Fe}^{3+}$ ions arranges themselves such that a $2 \mathrm{D}$ superstructure is formed within each individual W layer, largely owing to the stronger intralayer correlation ${ }^{24}$, as well as the interlayer correlation between $\mathrm{W}$ layers ${ }^{29-30}$. Here we attribute the phase transition of $\mathrm{Er}_{0.1} \mathrm{Yb}_{0.9} \mathrm{Fe}_{2} \mathrm{O}_{4}$ around $300 \mathrm{~K}$ to a $3 \mathrm{D}-2 \mathrm{D} \mathrm{CO}$ phase transition with increasing temperature, as marked by the 1372 and $1554 \mathrm{~cm}^{-1}$ peaks. Quantitatively, these Raman shift results provides explicit energy band information that the $2 \mathrm{D} C \mathrm{CO}$ is $170 \mathrm{meV}$ and $193 \mathrm{meV}$ higher that the 3D CO, respectively. Such an energy gap (Fig. 4c) exists at $300 \sim 400 \mathrm{~K}$.

Double 2D CO states with THz energy. In Fig. 2, the Raman peaks emerge at the temperature above $300 \mathrm{~K}$ are attributed to the $3 \mathrm{D}-2 \mathrm{D}$ phase transition. In the $2 \mathrm{D}$ CO state the atoms are closer than those in the 3D CO state, so the energy of the 2D CO is higher. Significantly we have observed two separate peaks, which indicated that the $2 \mathrm{D}$ $\mathrm{CO}$ is indeed composed of non-degenerate double states. The energies are $170 \mathrm{meV}(41.2 \mathrm{THz})$ and $193 \mathrm{meV}(46.6 \mathrm{THz})$ higher than that of the 3D CO state, respectively (Fig. 4c). We speculate that these non-degenerate double 2D CO states might come from two separate and different charge orderings within the W-layer: one comes from the nearest neighbors and the other comes from the next-to-nearest neighbors, thus forming charge orderings along different directions of the crystal lattice. Since collective CO or SO states are very sensitive to distances between atoms, this might explain the non-degeneracy of the double $\mathrm{THz}$ states. If this is true, then bi-directional ferroelectricity can be expected, which must coexist together (although not all the experimental means are sensitive to it).

Alternatively, these double 2D CO states might originate from a predicted fact ${ }^{9}$ that the $\mathrm{W}$-layer of the iron ions are asymmetric and composes of a $\mathrm{Fe}^{2+}$-rich layer and a $\mathrm{Fe}^{3+}$-rich layer simultaneously. Thus double long-range 2D CO develop within each sub-layer plane, but with non-degenerate energies. If the latter scenario is true, then our result provides direct experimental proof of the theoretical prediction of the bifurcated charge distribution and ordering within the W-layer in Ref. 9.

There is also a third possibility that this double $\mathrm{CO}$ state is originated from the doping of $\mathrm{Yb}$ atoms. The $\mathrm{CO}$ state reflects the properties of the Iron ions of the $\mathrm{W}$ layer, whereas the doping of $\mathrm{Er} / \mathrm{Yb}$ ions does not affect the charges of Iron ions directly. However, from Fig. 2 and 3, for the temperature range accommodating the double CO states (Fig. 2 \& 3), it can be seen that all the phonon modes (see below for discussion) show variations (in both frequency and intensity) with temperature, leading us to claim that the emergence of the double $\mathrm{THz} 2 \mathrm{D}$ CO is correlated to a structural softening. Note that both the phonon softening and the 2D CO states are associated to the varying lattice constants with temperature. The effect of doping might be conveyed by charge-lattice interactions. If this is true, then what we have found is a realization of generating double CO states by using chemical doping.

The observation of the double state of CO is unlikely due to slightly different states possibly due to the polycrystalline multi-domains, because we did not observe other multiple peaks and the two

Table 1 | Observed active modes in $\mathrm{Er}_{0.1} \mathrm{Yb}_{0.9} \mathrm{Fe}_{2} \mathrm{O}_{4}$ (at room temperature, if not specified)

\begin{tabular}{|c|c|c|c|c|c|c|}
\hline Mode Number & 1 & 2 & 3 & 4 & 5 & 6 \\
\hline $\begin{array}{l}\text { Frequency }\left(\mathrm{cm}^{-1}\right) \\
\text { Symmetry (Mode type) }\end{array}$ & $\begin{array}{c}129 \\
E_{u}^{\prime}(I R)\end{array}$ & $\begin{array}{c}216 \\
E_{g}^{\prime} \text { (Raman) }\end{array}$ & $\begin{array}{c}274 \\
A_{l g}{ }^{1} \text { (Raman) }\end{array}$ & $\begin{array}{c}360(78 \mathrm{~K}) \\
\text { (SO) }\end{array}$ & $\begin{array}{c}412 \\
\mathrm{~A}_{\mathrm{lg}}{ }^{2} \text { (Raman) }\end{array}$ & $\begin{array}{c}463 \\
\mathrm{E}_{\mathrm{g}}^{2} \text { (Raman) }\end{array}$ \\
\hline $\begin{array}{l}\text { Frequency }\left(\mathrm{cm}^{-1}\right) \\
\text { Symmetry (Mode type) }\end{array}$ & $\begin{array}{c}554 \\
E_{u}^{3}(I R)\end{array}$ & $\begin{array}{c}576 \\
\mathrm{E}_{\mathrm{g}}{ }^{3} \text { (Raman) }\end{array}$ & $\begin{array}{c}686 \\
\mathrm{~A}_{1 \mathrm{~g}}{ }^{3} \text { (Raman) }\end{array}$ & $\begin{array}{l}1372 \\
(\mathrm{CO} 1)\end{array}$ & $\begin{array}{l}1554 \\
\text { (CO2) }\end{array}$ & \\
\hline
\end{tabular}




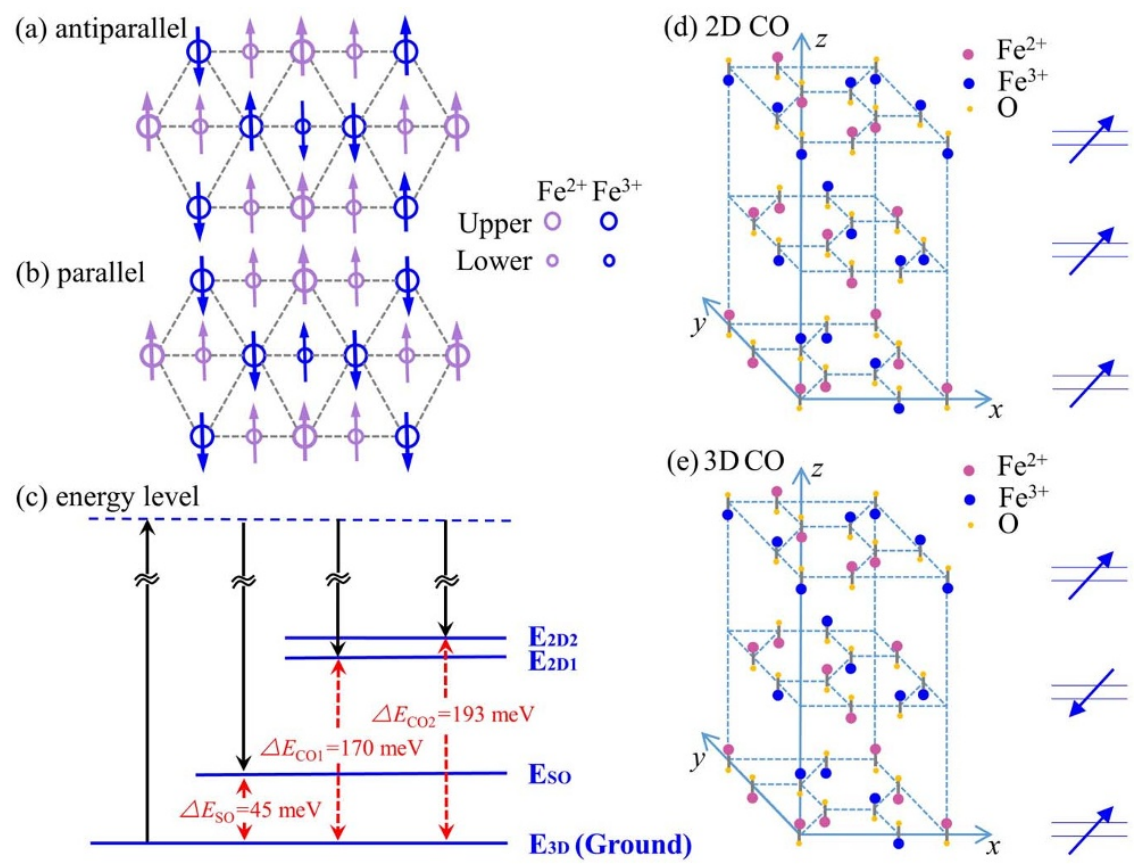

Figure 4 | Fine structure in energy for the orderings. The anti-parallel (a) and parallel (b) spin orderings in the material [Ref. 27]. (c) The schematic energy level for the double 2D COs, the SO, and the 3D CO with the energy gap values marked explicitly. Note the 3D CO level is very close to the ground state. Schematic lattice structures of the (d) 2D CO states and the (e) 3D CO state, where the polarization is marked by arrows explicitly on the right panel [Ref. 2].

Raman peaks are clearly separated. In any case, further investigation is needed to confirm the origin of the double CO states.

Spin Ordering State. Among the various investigations of the magnetic properties of $\mathrm{RFe}_{2} \mathrm{O}_{4}$, neutron scattering is most extensively used, with the initial identification of a ferrimagnetic ordering below $240 \mathrm{~K}$ in $\mathrm{LuFe}_{2} \mathrm{O}_{4}{ }^{31}$. Further neutron investigation with single crystal demonstrated that the ferrimagnetic spin ordering is of $3 \mathrm{D}$ nature ${ }^{22}$. Then the results of $\mathrm{X}$-ray absorption spectroscopy suggested that in the $\mathrm{W}$ layer the $\mathrm{Fe}^{2+}$ ions all align ferromagnetically and the $\mathrm{Fe}^{3+}$ ions experience triangular frustration, emerging two possible configurations each preserving a $1: 2$ ferrimagnetic ordering ${ }^{20}$. So far whether the two configurations are degenerate is not clear and no further evidence has been found to further identify the spin ordering thus formed. Particularly, no Raman scattering or other optical spectroscopy methods have been reported on observing such a spin ordering. Here in our result, when $T$ is below $210 \mathrm{~K}$, a new mode was unambiguously observed (mode 4 in Figs. 1-3), with a characteristic energy of $45 \mathrm{meV}(10.7 \mathrm{THz})$. Comparing with the available neutron and X-ray data we attribute this mode to the ferrimagnetic spin ordering of the system. Such a ground state of the spin structure is of long-range ordering nature and is usually elusive in a typical Raman scattering with linearly polarized probe to the center of the Brillion zone ${ }^{32-34}$. However, we have a polycrystalline sample immersed in the scatterings and breakings of the symmetry, which greatly relaxes the dipole transition restriction. In Fig. $4 \mathrm{a}-\mathrm{b}$, we show the suggested 3D spin ordering configuration of the system at temperatures below $210 \mathrm{~K}^{20}$. The fact that we have observed only one such spin ordering state implies that the two suggested configurations are either degenerate or nondegenerate with very small energy difference. From Fig. 3 it can be seen that this spin ordering state might be associated with the $\mathrm{E}_{\mathrm{g}}{ }^{3}$ mode phonon, where the interaction between them greatly modifies the phonon mode energy, especially at the low temperature regime.

Raman- and IR-active phonon modes. The $\mathrm{RFe}_{2} \mathrm{O}_{4}$ family crystals share a layered rhombohedral lattice structure (with space group
$\mathrm{R} \overline{3} \mathrm{~m})$ at room temperature. It consists of alternating $\mathrm{Fe}-\mathrm{O}$ doublelayers (W layer) and R-O layers (U layer) stacked along the $z$-axis. In the $\mathrm{W}$ layer two sheets of triangular corner-sharing $\mathrm{FeO}_{5}$ trigonal bipyramids constitute a layer of hexagonal $\mathrm{Fe}_{2} \mathrm{O}_{2.5}$, where the two sheets are shifted by $1 / 3 x$ in the $x y$ plane ${ }^{2}$. In the $\mathrm{U}$ layer the $\mathrm{R}_{2} \mathrm{O}_{3}$ atoms form a single flat layer instead. Such a $\mathrm{RFe}_{2} \mathrm{O}_{4}$ crystal structure preserves the center inversion symmetry. The lattice vibrations in $\mathrm{RFe}_{2} \mathrm{O}_{4}$ is constituted of six Raman-active modes and six infrared (IR)-active modes, i.e., $3 \mathrm{~A}_{1 \mathrm{~g}}+3 \mathrm{E}_{\mathrm{g}}$ for Raman and $3 \mathrm{~A}_{2 \mathrm{u}}+3 \mathrm{E}_{\mathrm{u}}$ for $\mathrm{IR}^{5-6}$. The displacement symmetry of the $\mathrm{A}_{1 \mathrm{~g}}$ mode is along the $c$-axis, whereby the R-Fe atoms vibrate in opposite directions along the $c$ axis. The displacement symmetry of the $\mathrm{E}_{\mathrm{g}}$ mode is along the $x y$ plane, whereby the atoms vibrate in opposite directions along to the $\mathrm{R}-\mathrm{O}$ and $\mathrm{Fe}-\mathrm{O}$ bonds, respectively. $\mathrm{Er}_{0.1} \mathrm{Yb}_{0.9} \mathrm{Fe}_{2} \mathrm{O}_{4}$ has been shown to have a similar structure to that of $\mathrm{LuFe}_{2} \mathrm{O}_{4}$ via $\mathrm{X}$-ray experiment ${ }^{7}$.

In conclusion, we have investigated the orderings and phase transition in the polycrystalline $\mathrm{Er}_{0.1} \mathrm{Yb}_{0.9} \mathrm{Fe}_{2} \mathrm{O}_{4}$ using Raman scattering. We for the first time found the double 2D CO states in the system and directly obtained the energy gap between the 2D and 3D CO states, which is at the $\mathrm{THz}$ range. Furthermore, a ferrimagnetic spin ordering mode was also observed, which has not been reported before in Raman scattering. Meanwhile two IR-active modes were also observed due to the symmetry breaking at the domain boundaries. Our investigation adds new feature to the fine structure of $\mathrm{RFe}_{2} \mathrm{O}_{4}$ system, which shines light to further investigations of other layered quantum materials.

\section{Methods}

Sample preparation. Our sample was synthesized using a conventional solid state reaction method. The details of the growth are described in Ref. 7. In addition, the sample surface was carefully polished mechanically before the Raman measurement. Due to the polycrystalline nature of the sample, the surface displays irregular dents. We measured only at the smooth part of the sample surface.

Variable-temperature Raman spectroscopy. All Raman data shown in this paper were obtained using a near-field con-focal spectrometer. The sample was kept in a continuous flow liquid nitrogen cryostat. The cryostat contains a heating element, making it possible to operate at temperatures ranging from 78 to $403 \mathrm{~K}$. Temperature 
fluctuation was kept as low as $0.1 \mathrm{~K}$. A $532 \mathrm{~nm}$ linearly polarized continuous wave laser beam with a power of $0.9 \mathrm{~mW}$ was used. An objective lens with $50 \times$ magnification was used to focus the laser light onto a $100 \mu \mathrm{m}$ slit of the spectrometer which has a spectral resolution of $2 \mathrm{~cm}^{-1}$. X-ray diffraction measurements were carried out to characterize the crystal lattice structure and phase purity. No impurity phase was observed, and the lattice constants are described in Ref. 7.

1. Ikeda, N. et al. Ferroelectricity from iron valence ordering in the charge-frustrated system $\mathrm{LuFe}_{2} \mathrm{O}_{4}$. Nature 436, 1136-1138 (2005).

2. Angst, M. et al. Charge order in $\mathrm{LuFe}_{2} \mathrm{O}_{4}$ : antiferroelectric ground state and coupling to magnetism. Phys. Rev. Lett. 101, 227601 (2008).

3. Xu, X. S. et al. Charge order dynamics, and Magnetostructural transition in multiferroic $\mathrm{LuFe}_{2} \mathrm{O}_{4}$. Phys. Rev. Lett. 101, 227602 (2008).

4. Christianson, A. D. et al. Three-Dimensional Magnetic Correlations in Multiferroic $\mathrm{LuFe}_{2} \mathrm{O}_{4}$. Phys. Rev. Lett. 100, 107601 (2008).

5. Harris, A. B. \& Yildirim, T. Charge and spin ordering in the mixed-valence compound $\mathrm{LuFe}_{2} \mathrm{O}_{4}$. Phys. Rev. B 81, 134417 (2010).

6. Hou, Y. et al. Temperature dependence of phonon spectra and structural characteristics in multiferroic $\mathrm{LuFe}_{2} \mathrm{O}_{4}$ system. J. Raman Spectroscopy 42, 1695-1700 (2011).

7. Wang, R. et al. Photoluminescence in electronic ferroelectric $\mathrm{Er}_{1-x} \mathrm{Yb}_{x} \mathrm{Fe}_{2} \mathrm{O}_{4}$. J. Appl. Phys. 108, 073507 (2010).

8. Zeng, L. J. et al. Nonlinear current-voltage behavior and electrically driven phase transition in charge frustrated $\mathrm{LuFe}_{2} \mathrm{O}_{4}$. Europhysics Letters 84, 57011 (2008).

9. Xiang, H. J. \& Whangbo, M.-H. Charge order and the origin of giant magnetocapacitance in $\mathrm{LuFe}_{2} \mathrm{O}_{4}$. Phys. Rev. Lett. 98, 246403 (2007).

10. Subramanian, M. A. et al. Giant room-temperature magnetodielectric response in the electric ferroelectric $\mathrm{LuFe}_{2} \mathrm{O}_{4}$. Advanced Materials 18, 1737-1739 (2006).

11. Rai, R. C., Delmont, A., Sprow, A., Cai, B. \& Nakarmi, M. L. Spin-charge-orbital coupling in multiferroic $\mathrm{LuFe}_{2} \mathrm{O}_{4}$ thin films. Appl. Phys. Lett. 100, 212904 (2012).

12. Kuepper, K. et al. Charge order, enhanced orbital moment, and absence of magnetic frustration in layered multiferroic $\mathrm{LuFe}_{2} \mathrm{O}_{4}$. Phys. Rev. B 80, 220409 (2009).

13. Wen, J., Xu, G., Gu, G. \& Shapiro, S. M. Magnetic-field control of charge structures in the magnetically disordered phase of multiferroic $\mathrm{LuFe}_{2} \mathrm{O}_{4}$. Phys. Rev. B 80, 020403(R) (2009).

14. Li, C. H. et al. Electrical control of magnetization in charge-ordered multiferroic. Phys. Rev. B 79, 172412 (2009).

15. Maruyama, T., Murakami, Y., Shido, D., Abe, N. \& Arima, T. Observations of charge-ordered and magnetic domains in $\mathrm{LuFe}_{2} \mathrm{O}_{4}$ using transmission electron microscopy. Phys. Rev. B 86, 054202 (2012).

16. Xu, X. S. et al. Lattice dynamics probe of charge order and antipolar bilayer stacking in $\mathrm{LuFe}_{2} \mathrm{O}_{4}$. Phys. Rev. B 82, 014304 (2010).

17. Vitucci, F. M. et al. Infrared study of the charge-ordered multiferroic $\mathrm{LuFe}_{2} \mathrm{O}_{4}$ Phys. Rev. B 81, 195121 (2010).

18. Glamazda, A. et al. Charge gap and charge-phonon coupling in $\mathrm{LuFe}_{2} \mathrm{O}_{4}$. Phys Rev. B 87, 144416 (2013).

19. Wu, W. et al. Formation of PancakelikeIsing Domains and Giant Magnetic Coercivityin Ferrimagnetic $\mathrm{LuFe}_{2} \mathrm{O}_{4}$. Phys. Rev. Lett. 101, 137203 (2008).

20. Ko, K. T. et al. Electronic Origin of Giant Magnetic Anisotropy in Multiferroic $\mathrm{LuFe}_{2} \mathrm{O}_{4}$. Phys. Rev. Lett. 103, 207202 (2009).

21. Wang, F., Kim, J. \& Kim, Y. J. Spin-glass behavior in $\mathrm{LuFe}_{2} \mathrm{O}_{4+\delta}$. Phys. Rev. B 80 024419 (2009)

22. Hearmon, A. J. et al. Helical scattering signatures of strain and electronic textures in $\mathrm{YbFe}_{2} \mathrm{O}_{4}$ from three-dimensional reciprocal-space imaging. Phys. Rev. B 85 , 014115 (2012)
23. Zhang, Y., Yang, H. X., Ma, C., Tian, H. F. \& Li, J. Q. Charge Order in $\mathrm{LuFe}_{2} \mathrm{O}_{4}$ Antiferroelectric Ground State and Coupling to Magnetism. Phys. Rev. Lett. 98, 247602 (2007)

24. Yamada, Y., Kitsuda, K., Nohdo, S. \& Ikeda, N. Charge and spin ordering process in the mixed-valence system $\mathrm{LuFe}_{2} \mathrm{O}_{4}$ : Charge ordering. Phys. Rev. B 62, 12167 (2000).

25. Tanaka, M., Siratori, K. \& Kimizuka, N. Mossbauer study of $\mathrm{RFe}_{2} \mathrm{O}_{4}$. Journal of the Physical Society of Japan 53, 760-772 (1984).

26. Zhang, Y., Yang, H. X., Ma, C., Tian, H. F. \& Li, J. Q. Charge-Stripe Order in the Electronic Ferroelectric LuFe $\mathrm{O}_{4}$. Phys. Rev. Lett. 98, 247602 (2007).

27. Mulders, A. M. et al. Direct Observation of Charge Order and an Orbital Glass State in Multiferroic $\mathrm{LuFe}_{2} \mathrm{O}_{4}$. Phys. Rev. Lett 103, 077602 (2009).

28. Ikeda, N. et al. Possibility of magnetoelectric effect in antiferromagnetic $\mathrm{RFe}_{2} \mathrm{O}_{4}$ Ferroelectic 161, 111-115 (1994).

29. Kudasov, Y. B. \& Maslov, D. A. Frustration and charge order in $\mathrm{LuFe}_{2} \mathrm{O}_{4}$. Phys. Rev. B 86, 214427 (2012)

30. Havlicek, M. et al. Indirect exchange interaction in fully metal-semiconductor separated single-walled carbon nanotubes revealed by electron spin resonance. Phys. Rev. B 86, 045402 (2012).

31. Iida, J. et al. Magnetization and spin correlation of two-dimensional triangular antiferromagnet $\mathrm{LuFe}_{2} \mathrm{O}_{4}$. Journal of the Physics Society of Japan 62, 1723-1735 (1993).

32. Cottam, M. G. \& Lockwood, D. J. Light scattering in magnetic solids. Wiley, New York (1986).

33. Zhao, J., Bragas, A. V., Lockwood, D. J. \& Merlin, R. Magnon squeezing in antiferromagnet: reducing the spin noise below the standard quantum limit. Phys. Rev. Lett. 93, 107203 (2004).

34. Zhao, J., Bragas, A. V., Lockwood, D. J. \& Merlin, R. Magnon squeezing in antiferromagnetic MnF2 and FeF2. Phys. Rev. B 73, 184434 (2006).

\section{Acknowledgments}

This work was supported by the National Basic Research Program of China (2012CB821402), the National Natural Science Foundation of China (11274372, 10974246, 51272277), the External Cooperation Program of the Chinese Academy of Sciences (GJHZ1403), the ACS Petroleum Research Fund (Grant 53401-UNI10), United States National Science Foundation (DMR-1337207, DMR-1410496), and the UNI Faculty Summer Fellowship.

\section{Author contributions}

J.Z. conceived and supervised the project. F.S., R.H. and J.Z. assigned the modes and plotted the figures. R.W. conducted the experiment. F.S. and J.Z. analyzed the $\mathrm{CO}$ and SO results. H.Y. provided the sample. C.A. prepared the table. J.Z. wrote the paper with input from all co-authors.

\section{Additional information}

Competing financial interests: The authors declare no competing financial interests.

How to cite this article: Sun, F. et al. Double Charge Ordering States and Spin Ordering State Observed in a $\mathrm{RFe}_{2} \mathrm{O}_{4}$ System. Sci. Rep. 4, 6429; DOI:10.1038/srep06429 (2014).

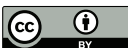

This work is licensed under a Creative Commons Attribution 4.0 International License. The images or other third party material in this article are included in the article's Creative Commons license, unless indicated otherwise in the credit line; if the material is not included under the Creative Commons license, users will need to obtain permission from the license holder in order to reproduce the material. To view a copy of this license, visit http://creativecommons.org/licenses/by/4.0/ 